\title{
Impaired phagocytosis and opsonisation towards group B streptococci in preterm neonates
}

\author{
Jan Källman, Jens Schollin, Claes Schalèn, Ann Erlandsson, Erik Kihlström
}

\begin{abstract}
Aims-To study the chemiluminescence response in polymorphonuclear leucocytes (PMNL) at different stages of maturity and the opsonic capacity of sera with defined titres of anti-capsular type III antibodies, after exposure to serotype III group B streptococci (GBS). The influence of GBS type III capsule expression on PMNL chemiluminescence response was also investigated.

Methods-Two clinical isolates of serotype III GBS and two serotype III reference strains which form isogenic variants with high and low amounts of capsule substance, respectively, were used. PMNL and sera were obtained from adult healthy blood donors, full term neonates, and preterm neonates.

Results-PMNL from premature infants showed a significantly lower chemiluminescence response $(p<0.0001)$ than the PMNL from adults and neonates, while the chemiluminescence response with adult, neonatal, and preterm sera gradually diminished. In the presence of a serum pool with a standardised complement value, raised $(>10 \mathrm{mg} / \mathrm{l})$, rather than low ( $<1.0 \mathrm{mg} / \mathrm{l})$ anti-III antibody titres induced a higher chemiluminescence response to the capsule expressing variant. When GBS were cultured at pH 5.0, the bacteria had a higher buoyant density, reflecting decreased expression of capsule substance compared with bacteria grown at pH 7.4. Concomitantly, there was a substantial increase in chemiluminescence response for all isolates cultured at the lower $\mathrm{pH}$, except for the capsule deficient mutant.

Conclusions-PMNL function and opsonic capacity are significantly impaired in neonates and correlate with maturation of the newborn child. The combined defect in cellular and humoral defences in preterm neonates may contribute to their increased susceptibility to GBS infection. Growth conditions for GBS, simulating different in vivo environments, greatly affect capsule expression and resistance to phagocytosis.

(Arch Dis Child Fetal Neonatal Ed 1998;78:F46-F50)
\end{abstract}

Keywords: phagocytosis; opsonisation; group B streptococci; chemiluminescence; capsule substance

Group B streptococci (GBS) are a major cause of invasive disease in neonates with rates of about 1 per 1000 live births. ${ }^{1}$ Low birthweight and prematurity increase the risk of fatal outcome. ${ }^{2}$ Serotype III accounts for about two thirds of GBS isolates associated with invasive neonatal disease. ${ }^{1}$ In a recently published study it was reported that serotype $\mathrm{V}$ has emerged as an important cause of invasive disease in neonates. ${ }^{3}$

Type specific GBS antibody titres, complement activity, and polymorphonuclear leucocyte (PMNL) function are major host defence mechanisms against neonatal GBS infection. For efficient ingestion and killing by PMNL, GBS serotype III strains must be opsonised with antibody and complement. ${ }^{4}$ Maximal opsonisation of serotype III strains depends on activation of classic complement pathway, and type specific antibodies. ${ }^{56}$ However, opsonisation also depends on alternative complement pathway activation. ${ }^{4}$ In general, resistance of GBS to phagocytosis is due to its capsule, a major virulence factor, of which the sialic acid determinants may act to impede the alternative way of complement activation. ${ }^{67}$

The two major receptors for $\mathrm{C} 3$ on the surface of human PMNL-CR1 and CR3-are critical to opsonophagocytosis of GBS. ${ }^{8}$ It has been suggested that the increased susceptibility of the neonate may depend on a deficient function of the alternative and classic complement pathways. $^{39}$

Abnormal function of PMNL is a major deficit that may increase the risk of bacterial infection in neonates. ${ }^{10}$ Previous studies have reported defective oxidative metabolic responses of PMNL from stressed and/or infected neonates while healthy term infants have normal phagocytic and bactericidal activities. ${ }^{11}{ }^{12}$ Preterm neonates have a decreased phagocytic capacity, as assessed by chemiluminescence response when exposed to opsonised zymosan, compared with term neonates and adults. ${ }^{10}$ Three weeks after birth this difference can no longer be seen, suggesting maturational changes in PMNL function. ${ }^{1013}$ However, previous studies have not compared the phagocytic capacity of PMNL from healthy, term, and preterm neonates towards GBS.

This study was undertaken to examine metabolic burst activity in different age related maturity stages of PMNL, and to determine the opsonic capacity of sera from premature and fullterm neonates in comparison with adults, when exposed to GBS. The influence of GBS type III capsule expression and anti-type III antibodies on PMNL chemiluminescence response was also studied. 


\section{Methods}

Two clinical isolates of serotype III GBS, VII and IX, obtained from the blood of neonates with clinical sepsis, were used together with two serotype III GBS reference strains. The reference strains U5 and U6 form isogenic variants in the sense that they were derived from the same original clinical isolate, and they express high and low amounts of capsule substance, respectively. Characteristics of these strains have been described before. ${ }^{14}$ The strains were cultured at $35-37^{\circ} \mathrm{C}$ in $4-6 \% \mathrm{CO}_{2}$ on haematin agar plates HEAMP; $4.25 \%$ Columbia II agar (Becton Dickinson Microbiology Systems, Cockeysville MD, USA), 3\% agar No 2 (Lab M, UK) and 8.5\% horse blood. Bacterial cells used for the chemiluminescence were collected from the plates, washed, and suspended in phosphate buffered saline solution (PBS), $\mathrm{pH} 7.4$ (0.14 M NaCL, $2.7 \mathrm{mM}$ KCL, $12 \mathrm{mM} \mathrm{Na}_{2} \mathrm{HPO}_{4}, 1.5 \mathrm{mM} \mathrm{KH} \mathrm{PO}_{4}, 0.9$ $\mathrm{mM} \mathrm{CaCl} 2$ and $0.49 \mathrm{mM} \mathrm{MgC}_{12}$ ). The bacteria were counted in a Bürker chamber and adjusted to $1.6-2.0 \times 10^{9} \mathrm{bacteria} / \mathrm{ml}$.

The bacterial strains were inoculated in $5 \mathrm{ml}$ Todd-Hewitt broth (Becton Dickinson Microbiology Systems) adjusted to $\mathrm{pH} 5.0$ and $\mathrm{pH}$ 7.4 , respectively, and cultured overnight with shaking at $35-37^{\circ} \mathrm{C}$ and $4-6 \% \mathrm{CO}_{2}$. From this overnight culture, $50 \mu \mathrm{l}$ was withdrawn, inoculated, and cultured overnight a second time at a different $\mathrm{pH}$, as described above. The strains were washed with $\mathrm{H}_{2} \mathrm{O}$ and pelleted by centrifugation at $2000 \times g$ for 10 minutes. The pellet was resuspended in $\mathrm{H}_{2} \mathrm{O}$ and the optical density at $620 \mathrm{~nm}$ was adjusted, corresponding to $3 \times 10^{9} \mathrm{bacteria} / \mathrm{ml}$. With an $\mathrm{L}$ shaped needle connected to a syringe, $0.5 \mathrm{ml}$ of the bacterial suspension was layered on top of a linear 1.02$1.12 \mathrm{~g} / \mathrm{ml} 3 \mathrm{ml}$ Percoll gradient (P-1644, Sigma Chemical Co. St Louis MO, USA). ${ }^{15}$ After centrifugation at $4^{\circ} \mathrm{C}$ for 2 hours at $3000 \times g$, the position of the bacterial band in the Percoll gradient was visually determined, and its density calculated from the sedimented distance.

Polymorphonuclear leucocytes were obtained from the venous blood of adult healthy blood donors (AG), cord blood from full term neonates (NG), or venous blood of premature (28-34 weeks of gestation) children (PG). The premature children had been admitted to the neonatal care unit at the Medical Centre Hospital, Örebro. The full term and premature neonates did not show any signs of infection or respiratory distress at the time when the blood samples were obtained. The blood was diluted with heparin and the PMNL were separated by Ficoll-Paque centrifugation (Pharmacia, Uppsala, Sweden), according to the method of Böyum, ${ }^{16}$ with minor modifications. After centrifugation the PMNL were washed in PBS with glucose $(1.25 \mathrm{~g} / \mathrm{l})$ and gelatine $(10 \mathrm{~g} / \mathrm{l})$ (PBSgg) to minimise the aggregation of cells and to prolong viability. ${ }^{17}$ After sedimentation in $1.4 \%$ dextran solution to separate PMNL and most of the erythrocytes, the remaining erythrocytes were lysed with $0.14 \mathrm{M}$ ammonium chloride. The cell suspension was then centrifuged at $400 \times g$, washed once, counted in a Bürker chamber, and resuspended in PBSgg to a concentration of 3-6 $310^{6}$ cells $/ \mathrm{ml}$. More than $95 \%$ of the cells were PMNL and previous staining with trypan blue of PMNL prepared by the same method have shown $95 \%$ viability. ${ }^{18}$

Pooled human serum from 10 healthy blood donors of blood group AB Rh+ (AS) was used in opsonisation experiments in combination with either AG, NG, or PG. Sera from 13 full term neonates (NS) were used in opsonisation experiments in combination with either AG or NG, and sera from five premature children (PS) were used together with AG. The pooled adult human serum had concentrations of $\mathrm{C} 3 / \mathrm{C} 4, \mathrm{IgG}, \operatorname{IgA}$, IgM as well as IgG subclasses within normal limits and showed normal complement dependent haemolytic activity with antibody coated sheep erythrocytes. ${ }^{19}$ The albumin concentration was normal (37 g/l). The acute phase proteins orosomucoid and C reactive protein were not increased.

The concentrations of C3c and GBS type specific antibodies in eight of the NS and four of the PS are shown in table $1 . \mathrm{C} 3 \mathrm{c}$ is a split product and reflects total $\mathrm{C} 3$ concentration and the reference range is $0.55-1.22 \mathrm{~g} / 1$. The C3c concentrations were determined by immunodiffusion (Boehringer Mannheim, Mannheim, Germany) and IgG antibodies against GBS capsular antigens were estimated in an enzyme immunoassay test. ${ }^{20}$

To examine the role of anti-type III antibodies in opsonisation, six sera with high $(>10$ $\mathrm{mg} / \mathrm{l})$ and six with low $(<1.0 \mathrm{mg} / \mathrm{l})$ concentrations of serotype III IgG antibodies were used. The sera were heat inactivated for 1 hour at $56^{\circ} \mathrm{C}$ and supplemented with equal amounts, $1: 1$, of serum, with no detectable titres of antibodies against the GBS serotypes Ia, Ib, II or III. With this external complement source the concentration of $\mathrm{C} 3 \mathrm{c}$ was $1.47 \mathrm{~g} / \mathrm{l}$ in each of the 12 sera.

A luminometer (LKB Wallac 1251, Turku Finland), connected to a Probyte midi computer, allowed up to 25 tests to be carried out in each experiment. A phagocytosis programme was used for measurements and calculations of data. To each test tube (LKB AB, Bromma, Sweden, No. 2174-086) the following was added: $200 \mu \mathrm{l}$ PBS, $100 \mu \mathrm{l}$ serum, $100 \mu \mathrm{l}$ PMNL, $50 \mu 110^{-5} \mathrm{M}$ Luminol (Sigma), and 50 $\mu \mathrm{l}$ of the bacterial suspension. The final ratio of bacteria:PMNL was about 200:1.

The chemiluminescence assays were run within 3 hours of collection of blood samples. The measurements were started within 2 minutes of adding the bacteria to the reaction. The assays were performed at $37^{\circ} \mathrm{C}$ with continuous shaking of the test tubes. Except for a few reactions with premature serum every sample was run in duplicate and measured at least every 194 seconds for about 25 minutes.

The chemiluminescence activity was recorded as a response curve over time and the peak value $(\mathrm{mV})$ was noted. The maximal rate constant was calculated as a function of chemiluminescence $(\mathrm{mV})$ and time (seconds) $-\mathrm{mV} /$ sec. To control the interassay variations, a reference reaction using GBS strain U6, pooled 


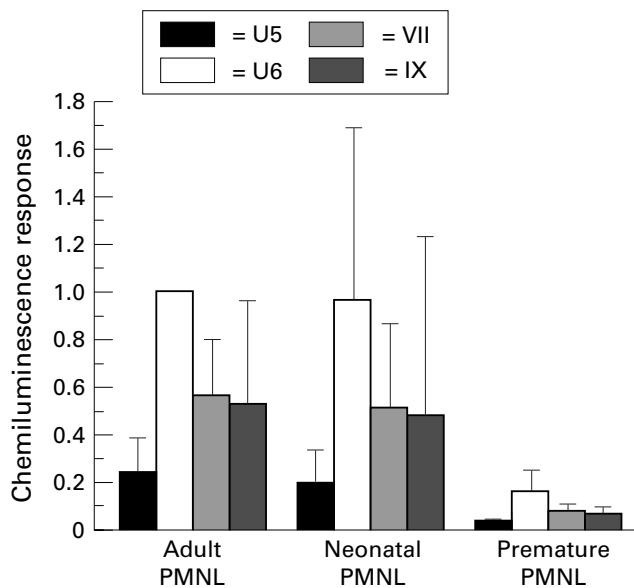

Figure 1 Chemiluminescence response ( $\mathrm{mV} /$ second) exposed to GBS of PMNL from adults, full term, and premature neonates. Pooled adult sera were used as a source of complement. Chemiluminescence response is expressed as ratios to response of strain U6 incubated with pooled human sera and adult blood donor PMNL. Bars represent mean (SD) of ratio of 4 to 11 individuals.

adult human serum (AS), and adult blood donor PMNL (AG) was included in each experiment. The CL response was expressed as the ratio between the test reaction and the reference reaction.

Statistical analysis for comparison between groups was performed using paired $t$ test. All analyses were performed using StatView.

\section{Results}

PHAGOCYTIC ACTIVITY OF PMNL FROM ADULTS, FULL TERM, AND PRETERM NEONATES

Phagocytosis has both a cellular component, exerted by PMNL, and a humoral component via antibodies and complement. Our aim was to discriminate between these two components and to study the contribution of each in the potential phagocytic defects against GBS in neonates. Therefore, the chemiluminescence response of PMNL from adults, full term, and preterm neonates exposed to GBS in vitro was recorded using a standardised humoral component. Pooled adult human sera with $1.6 \mathrm{mg} / \mathrm{l}$ of anti-serotype-III IgG antibodies and $1.00 \mathrm{~g} / \mathrm{l}$ of C3c was added to each set of PMNL. This antibody concentration is below the proposed protective level and $\mathrm{C} 3 \mathrm{c}$ is within normal values. ${ }^{5} 69$

Premature PMNL showed significantly lower chemiluminescence response $(\mathrm{p}<0.0001)$ when exposed to GBS than adult and full term PMNL (fig 1). This was true for all four GBS strains tested. However, there was no significant difference in chemiluminescence response of PMNL from adults and full term neonates. The capsule deficient mutant U6 induced the highest chemiluminescence response in all three sets of PMNLs-about four times the response of the capsule expressing variant $\mathrm{U} 5$.

OPSONIC ACTIVITY OF SERA

To standardise the cellular component of phagocytosis, PMNL from healthy adults were incubated in the presence of GBS, and with sera from adults, full term, and preterm

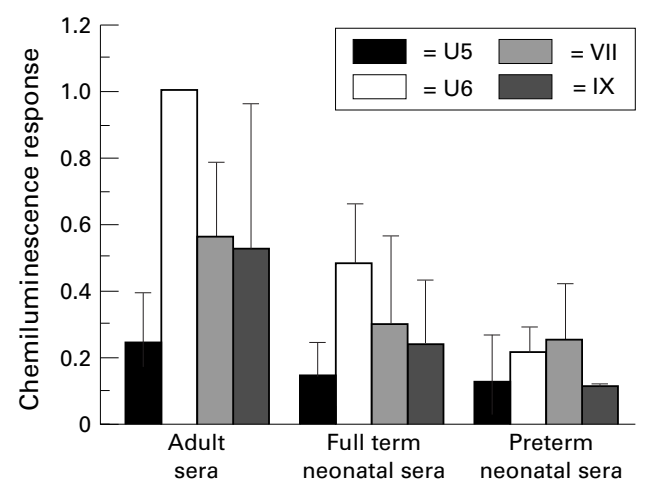

Figure 2 Comparison of chemiluminescence responses ( $\mathrm{mV} /$ second) of adult PMNL to GBS in the presence of sera from adult blood donors, full term, or preterm neonates. Bars represent mean (SD) of ratio of 2 to 13 individuals with at least 2 experiments carried out on each serum sample.

neonates. For all four GBS strains, the highest chemiluminescence response was recorded with the pooled adult sera (fig 2). However, in contrast to the situation with standardised humoral components, the chemiluminescence response gradually decreased in the presence of sera from full term neonates, and further still with preterm sera compared with the pooled adult sera. The greatest reduction in chemiluminescence response was observed for the capsule deficient variant U6. In the preterm neonatal sera the chemiluminescence response was similar for U6 and the capsule expressing variant U5. In contrast, in adult sera U6 induced a fourfold higher response than U5.

The concentration of $\mathrm{C} 3 \mathrm{c}$, and especially of anti serotype-III antibodies, varied between the sera used (table 1). However, the chemiluminescence response did not seem to vary between sera with high and low antibody titres of anti serotype-III antibodies. To further examine the role of serotype III antibodies, adult sera with high $(>10 \mathrm{mg} / \mathrm{l})$ and low $(<1.0$ $\mathrm{mg} / \mathrm{l}$ ) antibody titres were heat inactivated and supplemented with complement from a serum from a hypogammaglobulinaemic patient without detectable titres of type specific anti-GBS antibodies. The serotype III capsule expressing variant U5 induced a significantly higher $(p<0.0086)$ chemiluminescence response in PMNL, with sera containing high titres of serotype III antibodies compared with PMNL with low titres of antibodies (fig 3). In contrast, no difference in the PMNL response between sera with high and low antibody titres could be demonstrated when the capsule deficient variant U6 was studied. However, variant U6 in the presence of low antibody titres still induced a higher response than with variant $\mathrm{U} 5$ in the presence of high antibody titres.

EFFECT OF GBS CULTURE CONDITIONS AND CAPSULE EXPRESSION ON CHEMILUMINESCENCE Capsule expression of GBS significantly impairs phagocytosis, as assessed by chemiluminescence response (figs 1 to 3). Furthermore, growing GBS at $\mathrm{pH} 4.5$ inhibits capsule expression $^{21}$ and increases the buoyant density of the bacteria. ${ }^{15}$ When GBS strains U5, U6, VII and IX were grown at pH 5.0, they showed 
Table 1 IgG antibodies against GBS serotypes $I a, I b, I I$ and III in $m g / l$ and concentration of complement component $\mathrm{C} 3 \mathrm{c}$ in sera of adults, premature, and full term neonates

\begin{tabular}{|c|c|c|c|c|c|c|}
\hline Serum & No & $I A^{*}$ & $I B^{\star}$ & $I I^{\star}$ & $I I I^{\star}$ & $C 3 c t$ \\
\hline Adult & Pooled & 0.75 & 1.3 & 0.55 & 1.6 & 1.00 \\
\hline \multirow[t]{8}{*}{ Full term neonatal } & 20 & 0.5 & 0.7 & 0.5 & 0.8 & 1.06 \\
\hline & 23 & $<0.1$ & $<0.1$ & $<0.1$ & $>0.1$ & 0.95 \\
\hline & 24 & 0.8 & 5.9 & 1.7 & 10.2 & 1.17 \\
\hline & 28 & 1.3 & 1.2 & 0.7 & 2.6 & 1.06 \\
\hline & 29 & $<0.1$ & 0.6 & $<0.1$ & 0.6 & 0.84 \\
\hline & 50 & 0.8 & 3.3 & 1.2 & 5.4 & 0.84 \\
\hline & 57 & 0.8 & 4.3 & 1.6 & 6.6 & 1.23 \\
\hline & 61 & 0.7 & 0.6 & 0.5 & 0.6 & 1.06 \\
\hline \multirow{4}{*}{ Preterm neonatal } & 520 & $<0.1$ & $<0.1$ & 0.8 & 3.9 & 0.64 \\
\hline & 541 & 1.0 & 2.1 & 1.6 & $>10$ & 0.79 \\
\hline & 542 & $<0.1$ & $<0.1$ & $<0.1$ & $<0.1$ & 0.79 \\
\hline & 568 & 0.6 & 0.5 & $<0.1$ & 1.3 & 0.69 \\
\hline
\end{tabular}

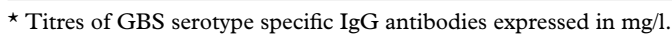

† Concentration of complement $\mathrm{C} 3 \mathrm{c}$ in $\mathrm{g} / \mathrm{l}$; reference range $0.55-1.22 \mathrm{~g} / 1$.

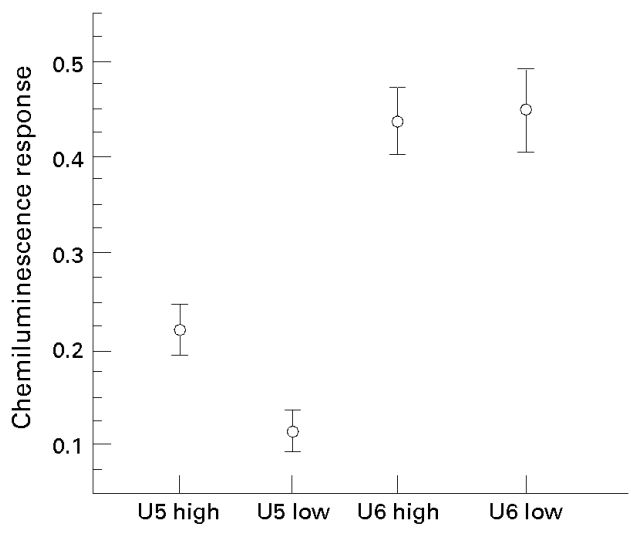

Figure 3 Chemiluminescence response ( $\mathrm{mV} /$ second) of adult PMNLs in the presence of sera from adult blood donors with high $(>10 \mathrm{mg} / \mathrm{l})$ or low $(<1.0 \mathrm{mg} / \mathrm{l})$ titres of GBS serotype III antibodies. GBS serotype III variants U5 and U6 express high and low amounts of capsule substance, respectively. Plots represent mean (SD) of 12 experiments.

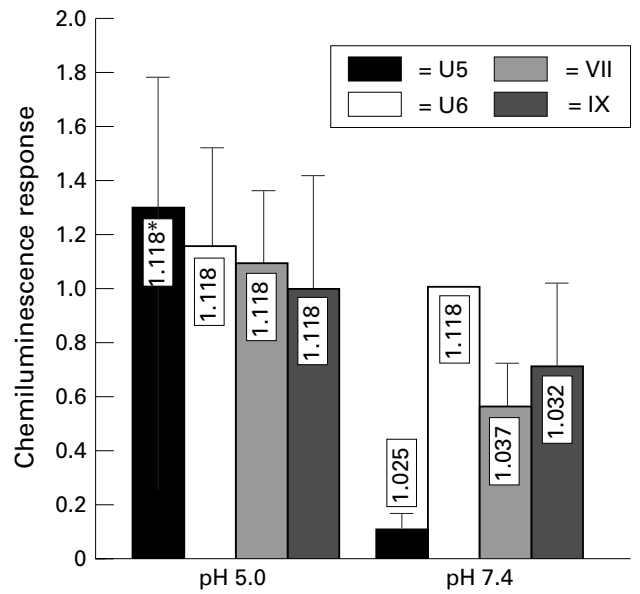

Figure 4 Chemiluminescence response ( $\mathrm{mV} /$ second) of adult blood from donor PMNLs and pooled human adult sera exposed to different GBS strains grown at $\mathrm{pH} 5$ and pH 7.4. The chemiluminescence response is expressed as the ration between the responses of strain U6 grown at $p H 7.4$. Bars represent mean (SD) of six experiments. ${ }^{\star}$ Density of strains, as determined by Percoll gradient centrifugation.

the same buoyant density of $1.118 \mathrm{~g} / 1$ in a Percoll gradient. There was also no difference in the chemiluminescence response of adult PMNL when exposed to these strains (fig 4). In contrast, when the strains were cultured at $\mathrm{pH}$ 7.4 and exposed to PMNL, there was a significant decrease in chemiluminescence response compared with growth at $\mathrm{pH} 5.0$ of the capsule expressing variants U5, VII, and IX $(\mathrm{p}<0.0021, \mathrm{p}<0.0023$, and $\mathrm{p}<0.056)$. Concomitantly, the density of these three variants decreased. The greatest reduction in chemiluminescence response and in buoyant density was observed for variant U5. For the capsule deficient variant $\mathrm{U} 6$, neither the chemiluminescence response nor the buoyant density was affected by growth at different $\mathrm{pH}$ values.

\section{Discussion}

GBS carriage is common in pregnant women, hence the ability of neonates to resist invasive GBS infections is of major importance for survival. As prematurity is a well known major risk factor for developing GBS disease, knowledge of the potential of neonates at various gestational ages to cope with GBS is of clinical interest.

We found that the chemiluminescence response of PMNL from premature infants compared with that of full term neonates was greatly depressed, whereas the response of the former did not differ significantly from that of healthy adults. PMNL from healthy newborns have been shown to exhibit normal phagocytosis of opsonised particles as well as particles that required no opsonization..$^{11} 2223$ On the other hand, stressed neonates and premature children generally show depressed chemiluminescence responses, and this deficient PMNL function in the premature infants may persist for up to two months after birth. ${ }^{12}{ }^{24}$ The mechanism for this is unknown, but one possibility might be a defective degranulation or low amount of enzymes in the primary and secondary granulae of PMNL. The PMNL of premature infants also show decreased chemotactic and adhesive capacities - the latter due to failure to upregulate surface expressed adhesive glycoproteins, which might also impair the ability to ingest GBS. ${ }^{1025}$ Furthermore, release of the chemoattractant interleukin-8 from neonatal monocytes stimulated with GBS is less than the release from adult monocytes. ${ }^{26}$ Another possibility might reside in rigid contractile cytoskeletal elements in premature PMNL with subsequent decreased internalisation into phagocytic vacuoles. ${ }^{10}$

In our study we were able to show an impaired chemiluminescence response of PMNL in the presence of neonatal and premature sera compared with that in adult sera. This difference between adult and full term serum samples might explain why term children might be prone to severe invasive GBS infections. Once again premature infants are disadvantaged as sera from these children show inferior opsonic capacity to those of full term children. This difference was most pronounced for the isolate $\mathrm{U} 6$ which is the isogenic variant with a low amount of capsular substance. The type III capsular polysaccharide inhibits the alternative pathway complement activation by preventing C3 deposition on the surface of GBS. ${ }^{27}$ Therefore, complement activation might be of major importance for opsonisation of GBS with low amounts of capsule substance. Furthermore, when serum was heat inactivated and no external complement was 
added, there were no chemiluminescence response. In the present study we did not investigate the role of different complement factors in the opsonisation of GBS strains. However, concentrations of $\mathrm{C} 3 \mathrm{c}$, which reflect total concentration of $\mathrm{C} 3$ as the pivotal protein site of convergence of the classic and alternative complement pathways, were within normal the range.

Decreased capsular substance, expressed as high buoyant density, could also be seen when GBS was cultured at pH 5.0, compared with that at $\mathrm{pH}$ 7.4. The substantial increase in chemiluminescence response for bacterial strains cultured at the lower $\mathrm{pH}$, except for the capsule deficient mutant, may reflect increased opsonic capacity by complement activation in the absence of the capsule. The expression of the capsular substance as dependent on the $\mathrm{pH}$ value might reflect a virulence factor for GBS. In genital tracts with a low $\mathrm{pH}$, the GBS will downregulate capsule substance expression. This might also lead to unmasking of surface exposed lipotheichoic acid, various surface proteins, or some other molecule(s) which may act as adhesions and thereby increase genital colonisation. ${ }^{28}{ }^{29}$ When GBS enter the bloodstream, due to the neutral $\mathrm{pH}$, the bacteria will switch on capsular expression conferring enhanced resistance to phagocytosis.

The relation between low concentrations of antibody to the capsular polysaccharide of type III GBS in maternal sera and the risk of invasive infection in neonates by GBS has been shown before. ${ }^{30}$ We have also shown that sera with high titres of antibody to capsule substance had a higher chemiluminescence response than sera with low titres and this might therefore protect against invasive infection.

In conclusion, the defect PMNL function and opsonic capacity against GBS in premature neonates calls for an aggressive clinical approach in these cases. The need for vaccine or highly concentrated type specific preparations for these children is therefore obvious. It is also extremely important to identify mothers at high risk of transmitting GBS to their offspring. The study also shows that the expression of capsular substance determines the ability to opsonise GBS successfully by complement or serotype III antibody. Finally, the expression of the capsular substance as dependent on different growth conditions may help us to understand better the pathogenic mechanisms leading to invasive GBS disease.

This study was supported by grants from the Research Committee of Örebro County, Örebro Medical Centre Research Foundation, King Gustaf V's 80-year Foundation, Börje Vahlin's Foundation and Sven Johanssons memorial Foundation.

We acknowledge the kind gift of GBS reference strains from Stellan Håkansson.

1 Baker CJ, Edwards MS. Group B streptococcal infections. In: Remington J, Klein JO, eds. Infectious diseases of the fetus and newborn infant. Philadelphia: WB Saunders, 1990:743811 .

2 Weisman LE, Stoll BJ, Cruess DF, Hall RT, Merenstein GB, Hemming VG, Fischer GW. Early-onset group B strepto- coccal sepsis; A current assessment. $\mathcal{F}$ Pediatr 1992;121:428-33.

3 Blumberg HS, Stephens DS, Modansky M, et al. Invasive group B streptococcal disease: The emergence of serotype V. F Infect Dis 1996;173:365-73.

4 Anderson DC, Edwards MS, Baker CJ. Luminol-enhanced chemiluminescence for evaluation of type III group B streptococcal opsonins in human sera. F Infect Dis 1980;141:370-81

5 Hill HR, Shigeoka AO, Hall T, Hemming VG. Neonatal cellular and humoral immunity to group B streptococci. Pediatrics 1979;64 (suppl):787-94.

6 Shigeoka AO, Hall RT, Allred CD, Hill HR. Role of antibody and complement in opsonization of group B streptococci. Infect Immun 1978;21:34-40.

7 Edwards MS, Kasper DL, Jennings HJ, Baker CJ, Nicholson-Weller A. Capsular sialic acid prevents activaNicholson-Weller A. Capsular sialic acid prevents activa-
tion of the alternative complement pathway by type III, tion of the alternative complement pathway by type
group B streptococci. F Immunol 1982;128:1278-83.

8 Smith CL, Baker CJ, Anderson DC, Edwards MS. Role of complement receptors in opsonophagocytosis of group B streptococci by adult and neonatal neutrophils. F Infect Dis
1990;162:489-95.

9 Davis CS, Vallota EH, Forrestal J. Serum complement levels in infancy: age-related changes. Pediatr Res 1979;13:1043-

10 Bektas S, Goetze B, Speer CP. Decreased adherence, chemotaxis and phagocytic activities of neutrophils from preterm neonates. Acta Paediatr Scand 1990;79:1031-8.

11 Shigeoka AO, Santos JI, HR Hill. Functional analysis of neutrophil granulocytes from healthy, infected and stressed neonates. F Pediatr 1979;95:454-60.

12 Shigeoka AO, Charette, Wyman, Hill HR. Defective oxidative metabolic responses of neutrophils from stressed tive metabolic responses of neutro
neonates. F Pediatr 1981;98:392-8.

13 Usmani SS, Schlessel JS, Concepcion GS, Kamran S.Dorner S. Polymorphonuclear leukocyte function in the preterm neonate: Effect of chronologic age. Pediatrics 1991;87:675-9.

14 Källman J, Schollin J, Håkansson S, Andersson A, Kihlström E. Adherence of group B streptococci to human endothelial cells in vitro. APMIS 1993;101:403-8.

15 Håkanson S, Granlund-Edstedt $M$, Sellin $M$, Holm SE. Demonstration and characterization of buoyant-density subpopulations of group B streptococcus type III. F Infect Dis 1990;161:741-6.

16 Böyum A. Separation of blood leukocytes, granulocytes and lymphocytes. Tissue Antigens 1974;4:269-74.

17 Halstensen A, Haneberg B, Glette J, Sandberg S, Solberg CO. Factors important for measurement of chemiluminescence production by polymorphonuclear leukocytes. $\mathcal{f}$ Immunol Methods 1986;88:121-8.

18 Fredlund H, Olcen P, Danielsson D. A reference procedure to study chemiluminescence induced in polymorphonuclear leukocytes by Neisseria meningitidis. APMIS 1988;96:941-9.

19 Mayer MM. Complement and complement fixation. Hemolytic assay of complement. In: Kabat, EA, Mayer MM, eds. Experimental immunochemistry. 2nd edn. Springfield, Illinois: Charles C Thomas, 1961:135-9.

20 Schalén C, Nilsson H-O, Zeeberg B. New EIA for GBS type-specific antibodies. In: Totolian AA, ed. Pathogenic streptococci: Present and future. St Petersburg, Lancer: 1994:357-8.

21 Sellin M, Håkansson S, Norgren M. Phase-shift of polysaccharide capsule expression in group B streptococci, type 995;18:401-15.

22 Forman ML, Stiehm ER. Impaired opsonic activity but normal phagocytosis in low-birth-weight infants. $N$ Engl 7 Med 1969;281:926-31.

23 McCracken JR, HF Eichenwald. Leukocyte function and the development of opsonic and complement activity in the neonate. Am F Dis Child 1971:121:120-6.

24 Driscoll MS, Thomas VL, Ramamurthy RS, Casto DT. Longitudinal evaluation of polymorphonuclear leukocyte chemiluminescence in premature infants. $\mathcal{f}$ Pediatr 1990;116:429-34.

25 Anderson DC, Freeman KB, Hughes BJ, Buffone GJ. Secretory determinants of impaired adherence and motility of neonatal PMNs. Pediatr Res 1985;19:257A.

26 Rowen JL, Smith W, Edwards MS. Group B streptococci elicit leukotriene B4 and interleukin-8 from human monocytes: Neonates exhibit a diminished response. $\mathcal{F}$ Infect Dis 1995;172:420-6.

27 Marques M, Kasper DL, Pangburn MK, Wessels MR. Prevention of $\mathrm{C} 3$ deposition by capsular polysaccharide is a virulence mechanism of type III group B streptococci. Infect Immun 1992;60:3986-93.

28 Bagg J, Poxton IR, Weir DM, Ross PW. Binding of type-III group B streptococci to buccal epithelial cells. $\mathcal{F} \mathrm{Med}$ Microbiol 1982;15:363-72.

29 Teti G, Tomasello F, Chiofalo MS, Orefici G, Mastroeni P. Adherence of group B streptococci to adult and neonatal epithelial cells mediated by lipoteichoic acid. Infect Immun 1987;55:3057-64.

30 Baker CJ, Kasper DL. Correlation of maternal antibody deficiency with susceptibility to neonatal group B streptococcal infection. N Engl f Med 1976;294:753-6. 